\title{
Number-theoretic Test Generation for Directed Rounding
}

\author{
Michael Parks \\ Java Technology Group \\ Sun Microsystems \\ 901 San Antonio Road \\ Palo Alto CA 94303 \\ Michael.Parks@sun.com
}

\begin{abstract}
We present methods to generate systematically the hardest test cases for multiplication, division, and square root subject to directed rounding, essentially extending previous work on number-theoretic floating-point testing to rounding modes other than to-nearest. The algorithms focus upon the rounding boundaries of the modes truncate, to-minusinfinity, and to-infinity, and programs based on them require little beyond exact arithmetic in the working precision to create billions of edge cases. We will show that the amount of work required to calculate trial multiplicands pays off in the form of free extra tests due to an interconnection among the operations considered herein. Although these tests do not replace proofs of correctness, they can be used to gain a high degree of confidence that the accuracy requirements as mandated by IEEE Standard 754 have been satisfied.
\end{abstract}

\section{Introduction}

We derive procedures to generate difficult cases for multiplication, division, and square root only for the directed rounding modes from IEEE 754. Similar tests for mode tonearest appear already in the U.C. Berkeley test suite [6]. The algorithms produce methodically rounding boundary cases for those modes via a number theory technique called Hensel lifting. While other methods based on factorization are possible, here we shall rely on one kind of construction to locate the data most likely to expose anomalies.

Our tests are intended for algorithms or hardware designs which are presumed reasonably correct. They are most appropriate for iterative schemes, such as Newton's iteration or Goldschmidt's algorithm for quotients and square roots (as discussed in Koren [4]), which typically compute a close approximation to the exact function value followed by a corrective step to get the last bit or two right. An erroneous routine based on such an algorithm is most likely to fail whenever the mathematical result lies close to a rounding boundary, which for any directed rounding mode is simply a floating-point number. We seek, then, test arguments $x$ and $y$ such that the function values $x y, x / y$, or $\sqrt{x}$ have several consecutive binary zeros or ones past the least significant bit: the longer that string, the stronger the test. But to find those test arguments, we need a trick to solve a sequence of congruence equations. A simple version of Hensel lifting and a few basic notes on IEEE floating-point arithmetic are stated in the next section.

Besides numerical malice, the strength of the material comes from a relationship that furnishes free extra tests (usually for division) which offsets the expense of a long recurrence to generate trial data for multiplication. Careful deduction of inequalities ensures that all operations are exact except those under examination. Furthermore, no multiprecision arithmetic is needed because every correctly rounded result falls out effortlessly as an upshot of the computation. After deriving conditions which determine judicious test data, we will analyze and explain the yields for the square root test and display a handful of examples of boundary cases, as well as the existence of a square root bug which our tests have uncovered.

\section{Hensel Lifting}

In order to find multiplicands whose product has a partly predetermined bit pattern, we shall use a technique from number theory to solve a certain type of congruence equation. In more general contexts Hensel lifting refers to a method to factor multivariate polynomials with integer coefficients, but for our purposes the following is sufficient.

THEOREM: Suppose $f$ is a polynomial with integer coefficients, $a$ is an integer such that $f(a) \equiv 0 \bmod 2^{j-1}$, and $f^{\prime}(a)$ is odd. Then $f(a-f(a)) \equiv 0 \bmod 2^{j}$. 
PROOF: Put $b=a-f(a)$, and consider $f(b)=$ $\sum_{0}^{N} c_{i} b^{i}=\sum_{0}^{N} c_{i}(a-f(a))^{i}$. Working modulo $2^{j}$, simplify $(a-f(a))^{i}$ to $a^{i}-i a^{i-1} f(a)$ since $f(a)^{i} \equiv 0 \bmod 2^{j}$ for $i \geq 2$. Thus $f(b) \equiv \sum_{0}^{N} c_{i} a^{i}-\sum_{1}^{N} i c_{i} a^{i-1} f(a) \equiv$ $f(a)\left(1-f^{\prime}(a)\right) \equiv 0 \bmod 2^{j}$ because $1-f^{\prime}(a)$ is even.

COROllaRY: Moreover, $f\left(b \bmod 2^{j}\right) \equiv 0 \bmod 2^{j}$.

PROOF: For any positive integer $j, f\left(b \bmod 2^{j}\right) \equiv$ $f(b) \bmod 2^{j}$, hence the claim.

The Hensel Theorem says that under mild conditions on $f$, any solution $a_{j-1}$ to the congruence $f(a) \equiv 0 \bmod 2^{j-1}$ can be lifted to a solution $a_{j}$ of $f(a) \equiv 0 \bmod 2^{j}$. In plainer language, if the last $j-1$ bits of $f\left(a_{j-1}\right)$ are zeros and $a_{j}=$ $a_{j-1}-f\left(a_{j-1}\right)$, then the last $j$ bits of $f\left(a_{j}\right)$ also are zeros, and in fact by the Corollary the last $j$ bits of $a_{j}$ will do. In the next section we recast the problem of finding critical test data for directed rounding of a product into the form of a congruence equation, and construct solutions to it using the Hensel Theorem. To ensure that the test is independent of the operation in question, we will determine conditions to restrict all intermediate terms in the algorithm to $n$ bits.

Besides test data, the algorithms also yield the correctly rounded results. To make them transparent, we list explicit formulas for the directed rounding modes prescribed by IEEE Standard 754 [1] using the floor and ceiling functions. For positive $x$ in the normalized range the rounding functions truncate, to-minus-infinity, and to-infinity are

$$
\begin{aligned}
\operatorname{trunc}(x) & =2^{e(x)-n+1}\left\lfloor 2^{n-1-e(x)} x\right\rfloor \\
\operatorname{minf}(x) & =2^{e(x)-n+1}\left\lfloor 2^{n-1-e(x)} x\right\rfloor \\
\inf (x) & =2^{e(x)-n+1}\left\lceil 2^{n-1-e(x)} x\right\rceil
\end{aligned}
$$

where the precision $n$ is understood and $e(x)=\left\lfloor\log _{2}(x)\right\rfloor$ is the exponent of $x$. We shall consider positive arguments since other tests follow from the symmetry relations

$$
\begin{aligned}
& \operatorname{trunc}(-x)=-\operatorname{trunc}(x) \\
& \operatorname{minf}(-x)=-\inf (x)
\end{aligned}
$$

and, as truncate and to-minus-infinity are identical from here on, the former needs no further mention. Our test arguments are scaled to locate all target products, quotients, and square roots inside one or two binades since checks near the edges of the exponent range can be deduced from the scaling properties

$$
\begin{aligned}
\operatorname{minf}\left(2^{m} x\right) & =2^{m} \operatorname{minf}(x) \\
\inf \left(2^{m} x\right) & =2^{m} \inf (x)
\end{aligned}
$$

Finally, the derivations are tailored to preclude overflow, underflow, and denormal numbers.

\section{Multiplication}

In this section we seek $n$-bit integers $x$ and $y$ in the range $\left[2^{n-1}+1,2^{n}-1\right]$ whose product is as close as possible to a directed rounding boundary. Since for such a case $e(x y)=$ $2 n-2$ or $2 n-1$, the product can be written as

$$
x y=2^{n-i} p \pm k
$$

where $i=0$ or $1, k$ is a small integer, and $p$ is an $n$-bit integer. The bigger $k$ is, the farther $x y$ is from a rounding boundary. We shall consider an odd $y$ (the other case will be solved later) and the minus sign, as the solution for the plus sign will spring from the derivation. Let $k$ be a small positive integer, and use Hensel lifting to solve

$$
f(x)=x y+k \equiv 0 \bmod 2^{n-i}
$$

for $x$ by solving the sequence of congruence equations

$$
x_{j} y+k \equiv 0 \bmod 2^{j}
$$

for $\left\{x_{j}\right\}_{1}^{n}$. To solve the first congruence is easy: take $x_{1}=$ 0 and $p_{1}=\frac{1}{2} k$ if $k$ is even, and $x_{1}=1$ and $p_{1}=\frac{1}{2}(y+$ $k$ ) if $k$ is odd. Proceed by mathematical induction: Given $f\left(x_{j-1}\right)=x_{j-1} y+k \equiv 0 \bmod 2^{j-1}$, define the integer $p_{j-1}$ by $x_{j-1} y+k=2^{j-1} p_{j-1}$. Since $f^{\prime}\left(x_{j-1}\right)=y$ is odd, the Hensel Theorem and Corollary imply $f\left(x_{j}\right)=$ $x_{j} y+k \equiv 0 \bmod 2^{j}$, where

$$
\begin{aligned}
x_{j} & =\left(x_{j-1}-f\left(x_{j-1}\right)\right) \bmod 2^{j} \\
& =\left(x_{j-1}-2^{j-1} p_{j-1}\right) \bmod 2^{j} \\
& =\left(x_{j-1}+2^{j-1} p_{j-1}\right) \bmod 2^{j} \\
& = \begin{cases}x_{j-1} & \text { if } p_{j-1} \text { is even } \\
x_{j-1}+2^{j-1} & \text { if } p_{j-1} \text { is odd }\end{cases}
\end{aligned}
$$

(Add $2{ }^{j} p_{j-1}$ to the second equation to get the third). We have established that

$$
x_{j} y+k=2^{j} p_{j}
$$

for an integer $p_{j}$, and so by construction every solution pair $\left(x_{j-1}, p_{j-1}\right)$ provides a successor, building $x_{n-1}$ and $x_{n}$ one bit at a time from right to left. Thus, given an odd $y$ in $\left[2^{n-1}+1,2^{n}-1\right]$ and small $k>0$, the full algorithm is

If $k$ is even, set $x_{1}=0$ and $p_{1}=\frac{1}{2} k$,
else set $x_{1}=1$ and $p_{1}=\frac{1}{2}(y+k)$

For $j=2, \ldots, n$, if $p_{j-1}$ is even set $x_{j}=x_{j-1}$ and $p_{j}=\frac{1}{2} p_{j-1}$, else set $x_{j}=2^{j-1}+x_{j-1}$ and $p_{j}=\frac{1}{2}\left(y+p_{j-1}\right)$

It is useful to bound the terms:

$$
x_{j} \leq 2^{j}-1 \quad \text { and } \quad 1 \leq p_{j} \leq y-1
$$


PROOFS: Since $x_{1} \leq 1$ and $x_{j} \leq 2^{j-1}+x_{j-1}$ the first claim holds by induction. The lower bound for $p_{j}$ is trivial, and the upper bound is also proved by induction, viz.: $p_{1}$ is either $\frac{1}{2} k$ or $\frac{1}{2}(y+k)$, so $p_{1} \leq \frac{1}{2}(y+k) \leq y-1$ because $k$ is a small integer. Given $p_{j-1} \leq y-1$, the recurrence ensures that $p_{j} \leq \frac{1}{2}\left(y+p_{j-1}\right) \leq y-\frac{1}{2}$, but that bound can be sharpened to $p_{j} \leq y-1$ since $p_{j}$ and $y$ are integers, hence the second claim.

Also, note that the possibility $x_{n-1}=0$ can be eliminated, since it implies that $k$ is an integer multiple of $2^{n-1}$, which does not correspond to any rounding boundary case. It remains to infer $x$ and $p$ such that $x y=2^{n-i} p \pm k$ from $x_{n-1}, x_{n}, p_{n-1}$, and $p_{n}$. Armed with $x_{n-1} y+k=$ $2^{n-1} p_{n-1}$ and bounds $1 \leq x_{n-1} \leq 2^{n-1}-1$ and $1 \leq$ $p_{n-1} \leq y-1$, two derived equations

$$
\begin{aligned}
\left(2^{n-1}+x_{n-1}\right) y & =2^{n-1}\left(y+p_{n-1}\right)-k \\
\left(2^{n}-x_{n-1}\right) y & =2^{n-1}\left(2 y-p_{n-1}\right)+k
\end{aligned}
$$

provide five situations such that $x y=2^{n-i} p \pm k$, but we must discard any for which $p$ does not fit into $n$ bits.

Consider the first equation. If $p_{n-1} \leq 2^{n}-1-y$, then both $x=2^{n-1}+x_{n-1}$ and $p=y+p_{n-1}$ are $n$-bit integers since $2^{n-1} \leq x \leq 2^{n}-1$ and the same for $p$. (Note that $2^{n}-1-y$ is computed exactly since $\left.y \geq 2^{n-1}+1\right)$. Since $x y=2^{n-1} p-k$, one pair of directed multiplication tests for correctly rounded products is

$$
\operatorname{minf}(x y)=2^{n-1}(p-1), \quad \inf (x y)=2^{n-1} p
$$

On the other hand if $p_{n-1} \geq 2^{n}-y$, then there are no worthwhile cases to pursue if $p_{n-1}$ is even, for then $y+$ $p_{n-1}$ is odd and exceeds $2^{n}$, and at least $n+1$ bits are needed to represent it. As long as $p_{n-1}$ is odd, put $x=$ $2^{n-1}+x_{n-1}$ and $p=\frac{1}{2}\left(y+p_{n-1}\right)$ to get $x y=2^{n} p-k$. (Note that the computation of $p$ is immune to roundoff error because $2^{n}+2 \leq y+p_{n-1} \leq 2 y-1 \leq 2^{n+1}-2$. Similar remarks apply henceforth). If $y+p_{n-1}>2^{n}$ check

$$
\operatorname{minf}(x y)=2^{n}(p-1), \quad \inf (x y)=2^{n} p
$$

but if instead $y+p_{n-1}=2^{n}$ check

$$
\operatorname{minf}(x y)=\left(2^{n}-1\right) p, \quad \inf (x y)=2^{n} p
$$

The latter case occurs infrequently, only when $x y$ barely undercuts a power of two.

The analysis for the second derived equation is similar. Suppose $p_{n-1} \geq 2 y-2^{n}+1$. From the bounds upon $x_{n-1}$ and $p_{n-1}$, both $x=2^{n}-x_{n-1}$ and $p=2 y-p_{n-1}$ are $n$-bit integers in the range $\left[2^{n-1}+1,2^{n}-1\right]$, and since $x y=2^{n-1} p+k$, the tests are

$$
\operatorname{minf}(x y)=2^{n-1} p, \quad \inf (x y)=2^{n-1}(p+1)
$$

Otherwise reject the case if $p_{n-1}$ is odd, for then $2 y-p_{n-1}$ is odd and exceeds $2^{n}$, and so does not fit into $n$ bits. Provided $p_{n-1}$ is even and at most $2 y-2^{n}$, put $x=2^{n}-x_{n}$ and $p=y-p_{n}$ so that $x y=2^{n} p+k$, and verify

$$
\operatorname{minf}(x y)=2^{n} p, \quad \inf (x y)=2^{n}(p+1)
$$

The smaller $y$ is, the more likely are $y+p_{n-1} \leq 2^{n}-1$ and $2 y-p_{n-1} \leq 2^{n}-1$, whereas the conditions $y+p_{n-1} \geq$ $2^{n}$ and $2 y-p_{n-1} \geq 2^{n}$ are more likely to apply if $y$ lies closer to $2^{n}-1$ than $2^{n-1}$.

The preceding test derivation can be generalized neatly to produce test cases if $y$ is an even integer in the range $\left[2^{n-1}+2,2^{n}-2\right]$. Write $y=2^{t} y^{\prime}$ where $t$ is the greatest power of 2 that divides $y$; then $y^{\prime}$ must be odd. Select an integer $k=2^{t} k^{\prime}$ where $k^{\prime}$ is an integer, and solve

$$
x y^{\prime}+k^{\prime}=2^{n-i-t} p
$$

by computing the solutions to

$$
x_{j} y^{\prime}+k^{\prime}=2^{j} p_{j}
$$

using the full algorithm up to step $n-t$, but first replace its $k$ by $k^{\prime}$ and $y$ by $y^{\prime}$. With $x_{n-1-t}, x_{n-t}, p_{n-1-t}$ and $p_{n-t}$ at hand, consider the equations

$$
\begin{aligned}
\left(2^{n-1}+x_{n-1-t}\right) y & =2^{n-1}\left(y+p_{n-1-t}\right)-k \\
\left(2^{n}-x_{n-1-t}\right) y & =2^{n-1}\left(2 y-p_{n-1-t}\right)+k
\end{aligned}
$$

Analogous to the case where $y$ is odd, there are five situations worth investigating. To distinguish them, the admissibility criteria for odd $y$ must be slightly recast, but the statements for verification are notationally unchanged and so are not repeated.

For the first derived equation, if $p_{n-1-t} \leq 2^{n}-1-y$, then set $x=2^{n-1}+x_{n-1-t}$ and $p=y+p_{n-1-t}$ so that $x y=2^{n-1} p-k$. Next if $y+p_{n-1-t} \geq 2^{n}$ and also $p_{n-1-t}$ and $y$ have the same parity, take $x=2^{n-1}+x_{n-1-t}$ and $p=\frac{1}{2}\left(y+p_{n-1-t}\right)$ to get $x y=2^{n} p-k$.

For the second equation, if $p_{n-1-t} \geq 2 y-2^{n}+1$, then set $x=2^{n}-x_{n-1-t}$ and $p=2 y-p_{n-1-t}$, hence $x y=$ $2^{n-1} p+k$. Last, if $p_{n-1-t}$ is even set $x=2^{n}-x_{n-t}$ and $p=y-p_{n-t}$ so that $x y=2^{n} p+k$ as wanted.

The preceding paragraphs actually generalize the case where $y$ is odd, for then $t=0$. And since it is plausible to test IEEE single precision exhaustively in a reasonable time it does no harm to require $x \geq y$ too.

Examples are shown in Table 1, using decimal form atop the conventional hexadecimal format with an underscore character for the binary point.

\section{Division}

Each valid pair of unequal multiplicands provides further tests for directed division tests at no cost. Given 


\begin{tabular}{|c|c|c|}
\hline$x$ & $y$ & $2^{n-i} x y$ \\
\hline 8388609 & 8388609 & $2^{23} \times 8388610.0000001 \ldots$ \\
$4 \mathrm{~b} 000001$ & $4 \mathrm{~b} 000001$ & $56800002 \_000002 \ldots$ \\
\hline 16777213 & 8388609 & $2^{23} \times 16777214.9999996 \ldots$ \\
$4 \mathrm{~b} 7 \mathrm{ffffd}$ & $4 \mathrm{~b} 000001$ & $56 \mathrm{fffffe} \_f f f f f a \ldots$ \\
\hline 8388611 & 8388609 & $2^{23} \times 8388612.0000003 \ldots$ \\
$4 \mathrm{~b} 000003$ & $4 \mathrm{~b} 000001$ & $56800004 \_000006 \ldots$ \\
\hline 12582911 & 8388610 & $2^{23} \times 12582913.9999997 \ldots$ \\
$4 \mathrm{~b} 3 \mathrm{fffff}$ & $4 \mathrm{~b} 000002$ & $56 \mathrm{c} 00001 \_f f f f f \ldots \ldots$ \\
\hline 12582914 & 8388610 & $2^{23} \times 12582917.0000004 \ldots$ \\
$4 \mathrm{~b} 400002$ & $4 \mathrm{~b} 000002$ & $56 \mathrm{c} 00005 \_000008 \ldots$ \\
\hline 13981013 & 8388611 & $2^{23} \times 13981017.9999998 \ldots$ \\
$4 \mathrm{~b} 555555$ & $4 \mathrm{~b} 000003$ & $56 \mathrm{~d} 55559 \_f f f f f e \ldots$ \\
\hline 11184811 & 8388611 & $2^{23} \times 11184815.0000001 \ldots$ \\
$4 \mathrm{~b} 2 \mathrm{a} a a a b$ & $4 \mathrm{~b} 000003$ & $56 a a a a a f \_000002 \ldots$ \\
\hline 11184810 & 8388611 & $2^{23} \times 11184813.9999997 \ldots$ \\
$4 \mathrm{~b} 2 \mathrm{a} a a a a$ & $4 \mathrm{~b} 000003$ & $56 a a a a a d \_f f f f f \ldots$ \\
\hline 13981014 & 8388611 & $2^{23} \times 13981019.0000002 \ldots$ \\
$4 \mathrm{~b} 555556$ & $4 \mathrm{~b} 000003$ & $56 \mathrm{~d} 5555 \mathrm{~b} \_000004 \ldots$ \\
\hline
\end{tabular}

Table 1. Boundary cases for multiplication

$x y=2^{n-i} p \pm k$, it is immediate that

$$
\frac{2^{n-i} p}{x}=y \pm \epsilon_{x} \quad \text { and } \quad \frac{2^{n-i} p}{y}=x \pm \epsilon_{y}
$$

for tiny $\epsilon_{x}=k / x$ and $\epsilon_{y}=k / y$. Since $x y=2^{n-i} p-k$ is a test case for multiplication, the corresponding tests for directed division are

$$
\begin{aligned}
& 2^{n-i} \operatorname{minf}(p / x)=y, \quad 2^{n-i} \inf (p / x)=y+1, \\
& 2^{n-i} \operatorname{minf}(p / y)=x, \quad 2^{n-i} \inf (p / y)=x+1
\end{aligned}
$$

With $x y=2^{n-i} p+k$, check for

$$
\begin{aligned}
& 2^{n-i} \operatorname{minf}(p / x)=y-1, \quad 2^{n-i} \inf (p / x)=y, \\
& 2^{n-i} \operatorname{minf}(p / y)=x-1, \quad 2^{n-i} \inf (p / y)=x
\end{aligned}
$$

The examples shown in Table 2 correspond to various rows in Table 1. Each has at least 21 consecutive like bits after the binary point; the first has 23 ones, which is provably maximal. These test data are far more likely to uncover a defective iterative division algorithm than random tests, and for very wide precisions no practical amount of random testing will stumble upon these patterns.

A complete derivation of test generation for square root is given in the next section. For the moment, note that if $x y \pm k=2^{n-i} p$ and $x=y$, then that multiplication test provides one test for directed division, and another for directed square root as follows. The derivation depends on the indicated sign and whether the exponent $n-i$ is even or odd. If the plus sign is used and $n-i$ is even, say $2 m$, then $x^{2}+k=2^{2 m} p$, and two square root tests are

$$
2^{m} \operatorname{minf}(\sqrt{p})=x, 2^{m} \inf (\sqrt{p})=x+1
$$

But if $n-i$ is odd, say $2 m+1$, then $x^{2}+k=2^{2 m+1} p$. Certainly $2 p$ is representable if $p$ is, and so

$$
2^{m} \operatorname{minf}(\sqrt{2 p})=x, 2^{m} \inf (\sqrt{2 p})=x+1
$$

Other tests associated with the equation $x^{2}-k=2^{n-i} p$ are obtained in a similar manner. For an even exponent, check

$$
2^{m} \operatorname{minf}(\sqrt{p})=x-1, \quad 2^{m} \inf (\sqrt{p})=x,
$$

and for odd exponent

$$
2^{m} \operatorname{minf}(\sqrt{2 p})=x-1, \quad 2^{m} \inf (\sqrt{2 p})=x
$$

For example, from Table 1, we know that $8388609^{2}-$ $1=2^{23} \times 8388610$, and so

$$
2^{11} \sqrt{2 \times 8388610}=8388608.99999994 \ldots
$$

Another case is $16777215^{2}-1=2^{24} \times 16777214$, with

$$
2^{12} \sqrt{16777214}=16777214.99999997 \ldots
$$

This trick can not be exploited to find division or square root boundary cases for mode to-nearest, because for that problem $x y$ (or $x^{2}$ ) must nearly equal the average of consecutive $n$-bit floating-point numbers, and exactly $n+1$ bits are required to represent that midpoint. 


\begin{tabular}{|c|c|c|}
\hline $2^{n-i} p$ & $x$ & $2^{n-2} p / x$ \\
\hline $2^{23} \times 8388610$ & 8388609 & $8388608.9999998 \ldots$ \\
\hline 56800002 & $4 \mathrm{~b} 000001$ & 4b000000_fffffe... \\
\hline $2^{23} \times 16777215$ & 16777213 & $8388609.0000001 \ldots$ \\
\hline 56 ffffff & $4 \mathrm{~b} 7 \mathrm{ffffd}$ & $4 \mathrm{~b} 000001 \_000003 \ldots$ \\
\hline $2^{23} \times 8388612$ & 8388611 & $8388608.9999996 .$. \\
\hline 56800004 & $4 \mathrm{~b} 000003$ & 4b000000_fffffa... \\
\hline $2^{23} \times 12582914$ & 12582911 & $8388610.0000001 \ldots$ \\
\hline $56 \mathrm{c00002}$ & $4 \mathrm{~b} 3 \mathrm{fffff}$ & $4 \mathrm{~b} 000002 \_000002 \ldots$ \\
\hline 12582913 & 12582910 & $8388610.0000003 \ldots$ \\
\hline $4 \mathrm{~b} 400001$ & $4 \mathrm{~b} 3 \mathrm{ffffe}$ & $4 \mathrm{~b} 000002 \_000005 \ldots$ \\
\hline $2^{n-i} p$ & $y$ & $2^{n-i} p / y$ \\
\hline $2^{23} \times 16777215$ & 8388609 & $16777213.0000003 \ldots$ \\
\hline 56 ffffff & $4 \mathrm{~b} 000001$ & 4b7ffffd_000005... \\
\hline $2^{23} \times 8388612$ & 8388609 & $8388610.9999996 \ldots$ \\
\hline 56800004 & $4 \mathrm{~b} 000001$ & 4b000002_fffffa... \\
\hline $2^{23} \times 12582914$ & 8388610 & $12582911.0000002 \ldots$ \\
\hline $56 c 00002$ & $4 \mathrm{~b} 000002$ & $4 b 3$ fffff_000003... \\
\hline $2^{23} \times 12582913$ & 8388610 & $12582910.0000004 \ldots$ \\
\hline $4 \mathrm{~b} 400001$ & $4 \mathrm{~b} 000002$ & $4 b 3$ ffffe_000007... \\
\hline
\end{tabular}

\section{Table 2. Boundary cases for division}

\section{Square Root}

This section establishes tests for square root under directed rounding, normalizing the problem so that the arguments to fall into two adjacent binades. We seek large integers $x$ so that $\sqrt{x}=z \pm \epsilon$ where $z$ is an $n$-bit integer and $\epsilon>0$ is tiny, so that $x=z^{2}-k$ for a small integer $k$ of either sign. Since $e(x)=2 n-2$ or $2 n-1$ and $x$ must be expressible using at most $n$ bits, we must have $x \equiv 0 \bmod 2^{n-i}$ where $i=0$ or 1 . Therefore

$$
z^{2} \equiv k \bmod 2^{n-i}
$$

and the smaller $|k|$ is, the closer $\sqrt{x}$ is to a directed rounding boundary. Suppose $k$ is odd (generalized later), then $z$ must be odd also, and consequently $k \equiv 1 \bmod 8$. To generate test integers, choose $k$ in $\{\ldots,-15,-7,1,9,17, \ldots\}$ and produce corresponding $z$ by solving the congruences

$$
z_{j}^{2} \equiv k \bmod 2^{j} \quad \text { for } j=1, \ldots, n
$$

Strictly speaking the Hensel Theorem in Section 2 is too specialized to bring the next recurrence to light, but it can be inferred from the discussion of " $p$-adic arithmetic" in Koblitz [3], and is easy to verify.

$$
\begin{aligned}
& \text { Set } z_{3}=1 \text { and let } z_{j}^{2}-k=2{ }^{j} R_{j} \\
& \text { For } j=4, \ldots, n,
\end{aligned}
$$

$$
\begin{aligned}
& \text { if } R_{j-1} \text { is even, set } z_{j}=z_{j-1} \text { and } R_{j}=\frac{1}{2} R_{j-1} \\
& \text { else set } z_{j}=2^{j-2}-z_{j-1} \text { and } \\
& \qquad R_{j}=2^{j-4}+\frac{1}{2}\left(R_{j-1}-z_{j-1}\right)
\end{aligned}
$$

A few relationships among the solutions to the recurrence borrowed from Kahan [2] will assist in the derivation of test cases and indicate the correctly rounded results. For any $k \equiv 1 \bmod 8$ the congruence $z^{2} \equiv k \bmod 2^{j}$ has infinitely many positive solutions. If $z_{j}$ is the smallest, then the four smallest solutions are

$$
\left\{z_{j}, 2^{j-1}-z_{j}, 2^{j-1}+z_{j}, 2^{j}-z_{j}\right\}
$$

lying in isometric integer ranges

$$
\begin{gathered}
{\left[1,2^{j-2}-1\right], \quad\left[2^{j-2}+1,2^{j-1}-1\right],} \\
{\left[2^{j-1}+1,3 \times 2^{j-2}-1\right], \quad\left[3 \times 2^{j-2}+1,2^{j}-1\right]}
\end{gathered}
$$

respectively. For $j=n-i$, whichever solutions $z$ satisfy $2^{2 n-1-i} \leq z^{2}-k \leq 2^{n-i}\left(2^{n}-1\right)$ provide difficult test integers $x=z^{2}-k$. Infer that the tests are

$$
\begin{array}{lll}
\operatorname{minf}(\sqrt{x})=z, & \inf (\sqrt{x})=z+1 & \text { if } k<0 \\
\operatorname{minf}(\sqrt{x})=z-1, & \inf (\sqrt{x})=z & \text { if } k>0
\end{array}
$$

The analysis of whether $z^{2}-k$ lies in an acceptable range splits into two cases. First, for $i=1$, use the recurrence to solve the congruence $z_{n-1}^{2} \equiv k \bmod 2^{n-1}$ to get four solutions $\left\{r_{1}, r_{2}, r_{3}, r_{4}\right\}=\left\{z_{n-1}, 2^{n-2}-z_{n-1}, 2^{n-2}+\right.$ 
$\left.z_{n-1}, 2^{n-1}-z_{n-1}\right\}$. It turns out that no such solution is large enough for $x$ to lie in $\left[2^{2 n-2}, 2^{n-1}\left(2^{n}-1\right)\right]$, but there is a remedy. We will show that $s_{1}=2^{n-1}+r_{1}$ leads to a valid test integer, as does $s_{2}=2^{n-1}+r_{2}$ if it is small enough. Note $s_{1}^{2} \equiv s_{2}^{2} \equiv k \bmod 2^{n-1}$ as required.

LEMMA: $s_{1}$ is admissible, and $s_{2}$ is admissible if $s_{2}<$ $c_{1}=\sqrt{2^{2 n-1}-2^{n-1}}$

PROOFS: Assume that $0<\epsilon \leq 1 / 2^{5}$ so that $|k| \leq 2^{n-4}$ and $\sqrt{x}$ has at least four consecutive zeros or ones past the binary point. For all $k$ of interest, $s_{1}^{2}-k \geq\left(2^{n-1}+1\right)^{2}-$ $2^{n-4}>2^{2 n-2}$, and $s_{1}<2^{n-1}+2^{n-3}$, so $s_{1}^{2}-k<\left(2^{n-1}+\right.$ $\left.2^{n-3}\right)^{2}+2^{n-4}<2^{n-1}\left(2^{n}-1\right)$. Since $s_{2}>s_{1}$ we have $s_{2}^{2}-k>2^{2 n-2}$. We must also decide whether $s_{2}^{2}-k \leq$ $2^{2 n-1}-2^{n-1}$, or equivalently whether $s_{2}^{2}-2^{2 n-1}+2^{n-1} \leq$ $k$. The parabola $k(r)=r^{2}-2^{2 n-1}+2^{n-1}$ is extremely steep on the domain of those $r$ for which $|k| \leq 2^{n-4}$, so steep that comparing $s_{2}$ to the positive zero $c_{1}$ of $k(r)$ is enough to determine whether $s_{2}^{2}-k \leq 2^{n-1}\left(2^{n}-1\right)$.

In brief, if $k$ is not too large then the solutions of the congruence can be checked quickly for validity. The same is true for the second case, $i=0$, for which we use the recurrence to solve $z_{n}^{2} \equiv k \bmod 2^{n}$ for $\left\{r_{1}, r_{2}, r_{3}, r_{4}\right\}=$ $\left\{z_{n}, 2^{n-1}-z_{n}, 2^{n-1}+z_{n}, 2^{n}-z_{n}\right\}$. We will show that $r_{4}$ is in $\left[2^{2 n-1}, 2^{n}\left(2^{n}-1\right)\right]$, as is $r_{3}$ if large enough.

LEMMA: $r_{4}$ is always admissible, and $r_{3}$ is admissible if $r_{3} \geq c_{0}=2^{n-1 / 2}$

PROOFS: For all $k$ of interest, $r_{4}^{2}-k \geq\left(3 \times 2^{n-2}+1\right)^{2}-$ $2^{n-4}>2^{2 n-1}$, and $r_{4}^{2}-k \leq\left(2^{n}-1\right)^{2}+2^{n-4}<2^{n}\left(2^{n}-1\right)$. Next, $r_{3}^{2}-k<r_{4}^{2}-k<2^{n}\left(2^{n}-1\right)$, so $r_{3}$ is never too large. It remains to determine whether $r_{3}^{2}-k \geq 2^{2 n-1}$, or equivalently $k \leq r_{3}^{2}-2^{2 n-1}$. But the $r$-coordinates of the intersection of the parabola $k(r)=r^{2}-2^{2 n-1}$ with lines $k= \pm 2^{n-4}$ hardly differ, so it suffices to compare $r_{3}$ to the zero $c_{0}$ of $k(r)$.

From here on, let $r_{3}$ and $r_{4}$ refer to the case $i=0$ only. The recurrence always provides two integers, one for $i=1$ and another for $i=0$. But more than three is impossible:

THEOREM: No $k$ produces four test integers

PROOF: If $R_{n-1}$ is even, then $z_{n}=z_{n-1}$, so $r_{3}=$ $2^{n-1}+z_{n}=2^{n-1}+z_{n-1}=s_{1}$. Hence $r_{3}^{2}-k=s_{1}^{2}-k \leq$ $2^{n-1}\left(2^{n}-1\right)<2^{2 n-1}$, and therefore $r_{3}$ is too small to produce a test integer. If $R_{n-1}$ is odd, then $z_{n}=2^{n-2}-z_{n-1}$, so $r_{3}=2^{n-1}+2^{n-2}-z_{n-1}=s_{2}$. If also $s_{2}$ is admissible, then $r_{3}^{2}-k \leq 2^{n-1}\left(2^{n}-1\right)<2^{2 n-1}$.

Either two or three integers are produced for each $k$. If distributed evenly within its range, solution $s_{2}$ would be valid about $\left(c_{1}-5 \times 2^{n-3}\right) / 2^{n-3} \approx 4 \sqrt{2}-5=65.7 \%$ of the time, and $r_{3}$ would be valid about $\left(3 \times 2^{n-2}-\right.$

\begin{tabular}{|c|c|}
\hline $2^{n-i} x$ & $\sqrt{2^{n-i} x}$ \\
\hline $2^{23} \times 8388610$ & $8388608.99999994 \ldots$ \\
\hline 56800002 & $4 \mathrm{~b} 000000$ _ffffffo... \\
\hline $2^{24} \times 16777214$ & $16777214.99999997 \ldots$ \\
\hline 577 ffffe & $4 b 7$ ffffe_ffffef $7 \ldots$ \\
\hline $2^{23} \times 8388612$ & $8388609.9999997 \ldots$ \\
\hline 56800004 & 4b000001_fffffe... \\
\hline $2^{24} \times 16777212$ & $16777213.9999998 \ldots$ \\
\hline $577 \mathrm{ffffc}$ & $4 \mathrm{~b} 7$ ffffd_ffffffd... \\
\hline $2^{23} \times 11946704$ & $10010805.0000003 \ldots$ \\
\hline $56 \mathrm{~b} 64 \mathrm{ad} 0$ & 4b18c0b5_000005... \\
\hline $2^{23} \times 14321479$ & $10960715.0000003 \ldots$ \\
\hline 56 da 8747 & 4b273f4b_000005 ... \\
\hline $2^{24} \times 13689673$ & $15155019.0000002 \ldots$ \\
\hline $5750 e 349$ & 4b673f4b_000003... \\
\hline $2^{23} \times 8388614$ & $8388610.9999994 \ldots$ \\
\hline 56800006 & $4 \mathrm{~b} 000002$ ffffff7... \\
\hline $2^{24} \times 16777210$ & $16777212.9999997 \ldots$ \\
\hline $577 \mathrm{ffffa}$ & $4 \mathrm{~b} 7 \mathrm{ffffC}$ _fffffb... \\
\hline $2^{23} \times 10873622$ & $9550631.0000007 \ldots$ \\
\hline $56 a 5 e b 16$ & 4b11bb27_00000d... \\
\hline
\end{tabular}

Table 3. Boundary cases for square root

$\left.c_{0}\right) / 2^{n-2}=3-2 \sqrt{2}=17.2 \%$ of the time. For odd $k$, our program corroborates those figures, and averages 2.828 (looks suspiciously like $2 \sqrt{2}$ ) integers per $k$ when testing double precision. A similar analysis ought to explain the percentages in the last section of Kahan [2].

At any rate, each trial case can be computed economically. In the chart below, each parenthesized expression fits into $n$ bits, since $\left|R_{j}\right|<2^{j-4}$ and $z_{j}<2^{j-2}$ for all $j$ large enough, again, as proved in Kahan's note.

For solution $z \ldots \quad$ compute $x=z^{2}-k$ via $\ldots$

$$
\begin{array}{ll}
2^{n-1}+z_{n-1} & 2^{n-1}\left(2^{n-1}+\left(R_{n-1}+2 z_{n-1}\right)\right) \\
3 \times 2^{n-2}-z_{n-1} & 2^{n-1}\left(9 \times 2^{n-3}+\left(R_{n-1}-3 z_{n-1}\right)\right) \\
2^{n-1}+z_{n} & 2^{n}\left(2^{n-2}+\left(R_{n}+z_{n}\right)\right) \\
2^{n}-z_{n} & 2^{n}\left(2^{n}+\left(R_{n}-2 z_{n}\right)\right)
\end{array}
$$

As for multiplication, there is a generalization to find more square root test cases if $k$ is an even integer. If there are any test integers to find for even $k$, then the corresponding $z$ must be even as well, and so $k$ must be divisible by 4 to begin with. Write $k=4^{t} k^{\prime}$ where $t$ is the greatest power of 4 which divides $k$, and let $z=2^{t} z^{\prime}$. Both $k^{\prime}$ and $z^{\prime}$ are odd, so we must have $k^{\prime} \equiv 1 \bmod 8$. To obtain trial cases, solve the congruences $z_{j}^{\prime 2} \equiv k^{\prime} \bmod 2^{n-i-2 t}$ using the lifting algorithm up to step $n-i-2 t$, but first replace its $z_{j}$ by $z_{j}^{\prime}$ and $k$ by $k^{\prime}$. Then set $z_{n-1}=2^{t} z_{n-1-2 t}^{\prime}$ and $z_{n}=2^{t} z_{n-2 t}^{\prime}$ to solve the congruences $z^{2} \equiv k \bmod 2^{n-i}$. 


\begin{tabular}{|c|c|c|}
\hline $2^{n-i} x$ & Expected $\operatorname{minf}\left(\sqrt{2^{n-i} x}\right)$ & Computed $\operatorname{minf}\left(\sqrt{2^{n-i} x}\right)$ \\
\hline $2^{53} \times 8732221479794286$ & 8868644699447394 & 8868644699447395 \\
\hline $468 \mathrm{f} 05 \mathrm{e} 8 \mathrm{bf} 67366 \mathrm{e}$ & $433 f 81 f_{C} 40 f 32062$ & $433 f 81$ fc $40 f 32063$ \\
\hline $2^{52} \times 8550954388695124$ & 6205648636410980 & 6205648636410981 \\
\hline $467 e 610 c 36 d 42854$ & $43360 c 012 a 92 f_{C} 64$ & $43360 c 012 a 92 f c 65$ \\
\hline $2^{52} \times 7842344481681754$ & 5942960515215712 & 5942960515215713 \\
\hline $467 \mathrm{bdc} 921 \mathrm{~d} 09715 \mathrm{a}$ & $43351 d 17526 \mathrm{c} 7160$ & $43351 d 17526 c 7161$ \\
\hline $2^{52} \times 5935035262218600$ & 5170011856404048 & 5170011856404049 \\
\hline $467515 e 21488 \mathrm{~b} 168$ & 43325 e19302f7e 50 & $43325 e 19302 f 7 e 51$ \\
\hline $2^{52} \times 5039650445085418$ & 4764091504847932 & 4764091504847933 \\
\hline 4671 e 7890 e 924 aea & 4330 ecea $7 d d 2$ ec $3 c$ & 4330 ecea $7 d d 2 e c 3 d$ \\
\hline $2^{52} \times 5039721545366078$ & 4764125111052576 & 4764125111052577 \\
\hline $4671 e 7999 c 7 a 7 a 3 e$ & 4330 ecf 250 e 8 e 920 & 4330 ecf 250 e 8 e 921 \\
\hline $2^{52} \times 8005963117781324$ & 6004635918520114 & 6004635918520115 \\
\hline $467 \mathrm{c} 71618 \mathrm{bb} 8614 \mathrm{c}$ & $4335552 f 3 e e d c f 32$ & 4335552 f3eedcf33 \\
\hline $2^{52} \times 6703494707970582$ & 5494529667669144 & 5494529667669145 \\
\hline $4677 \mathrm{~d} 0 \mathrm{cafcd} 28216$ & 4333853 ee10c9c98 & 4333853 ee10c9c99 \\
\hline $2^{52} \times 8010323124937260$ & 6006270743197038 & 6006270743197039 \\
\hline $467 \mathrm{c} 7558 \mathrm{~b} 065 \mathrm{e} 22 \mathrm{c}$ & $433556 \mathrm{abe} 212 \mathrm{~b} 56 \mathrm{e}$ & $433556 \mathrm{abe} 212 \mathrm{~b} 56 \mathrm{f}$ \\
\hline $2^{53} \times 8010776873384260$ & 8494390118415981 & 8494390118415982 \\
\hline $468 c 75 c 255$ e $9 b 944$ & $433 e 2 d 9 a 51977 e 6 d$ & $433 e 2 d 9 a 51977 e 6 e$ \\
\hline
\end{tabular}

Table 4. Erroneous double precision square roots in an early hyperSPARC

Admissible solutions are $s_{1}=2^{n-1}+z_{n-1}$ and possibly $s_{2}=2^{n-1}+2^{n-2}-z_{n-1}$ (for $i=1$ ), along with $r_{4}=$ $2^{n}-z_{n}$ and possibly $r_{3}=2^{n-1}+z_{n}$ (for $i=0$ ). At most three test integers are computed using a modified version of the chart; just replace each instance of $R_{n-1}$ by $R_{n-1-2 t}$ and $R_{n}$ by $R_{n-2 t}$.

Finally, note that if $t=0$ then the previous two paragraphs handle the case where $k$ is odd, so the generalized algorithm runs through the set

$$
\{\ldots,-28,-23,-15,-7,1,4,9,16,17,25,33,36, \ldots\}
$$

of integers $k=4^{t} k^{\prime}$ with $t \geq 0$ and $k^{\prime} \equiv 1 \bmod 8$. About one-sixth of all integers in any large symmetric range qualify, empirically yielding 2.67 test integers per $k$ on average.

Some examples are shown in Table 3 . The first two have 24 consecutive ones past the binary point, and each square root differs from the nearest 24-bit integer by less than $2^{-20}$.

A program based on these techniques revealed almost instantly erroneous double precision square roots on an early prototype hyperSPARC chip. The first ten errors found for mode to-minus-infinity are listed in Table 4.

\section{Conclusion}

We developed methods to generate in descending order of difficulty test data for three fundamental operations by using lifting techniques from number theory. They should be used to reinforce suspicion that an algorithm or hardware design conforms to the expectations for directed rounding imposed by the IEEE Standard for binary arithmetic.

We rigorously established that no intermediate operations depend upon rounded sums, differences, or products, and therefore programs to implement these algorithms should require very little beyond exact addition and subtraction, along with multiplication by powers of two and small constants. The correctly rounded results for validation are apparent without extra work, and further tests can be finessed from each multiplication case at no cost. Moreover, we have demonstrated that extreme cases on both sides of at least a few rounding boundaries always exist; any accuracy claims for directed rounding algorithms to the effect that "the worst case error is 0.998 units in the last position of the computed result" (or some smaller figure) are invalid.

The discussion herein generalizes previous work on deriving these kinds of trials (in Kahan [2] and Tang [5]) which did not tackle the problem for even multiplicands, divisors, and radicands. Sufficiently adequate properties of the rounding modes have been put forth to construct tests for sign combinations and function values at the edges of the exponent range. To conclude, these methods give rise to the numerically rarest of situations, making errors in these directed rounding operations practically inexcusable. 


\section{Acknowledgments}

Doug Priest and Alex Liu of Sun Microsystems offered many valuable remarks as these notes and programs were in progress. I am indebted to Professor William Kahan, K. C. Ng of Sun Microsystems, and P. T. Peter Tang, whose previous work led to this note, and also to my thesis advisor, Professor Beresford N. Parlett, for insightful comments.

The reviews of the referees were appreciated.

\section{References}

[1] IEEE 754-1985 Standard for Binary Floating-Point Arithmetic, Institute for Electrical and Electronics Engineers, New York, 1985.

[2] William Kahan, A Test for Correctly Rounded SQRT, http: //www.cs.berkeley.edu/ wkahan/ sQRTest.ps.

[3] Neal Koblitz, $p$-adic Numbers, $p$-adic Analysis, and ZetaFunctions, Graduate Texts in Mathematics, volume 58, Springer-Verlag, 1984.

[4] Israel Koren, Computer Arithmetic Algorithms, Prentice Hall, 1993.

[5] Ping Tak Peter Tang, Testing Computer Arithmetic by Elementary Number Theory, Preprint MCS-P84-0889, Mathematics and Computer Science Division, Argonne National Laboratory, August 1989.

[6] The U.C. Berkeley test suite, available from Netlib, http://www. netlib.org/fp/ucbtest.tar.gz. 